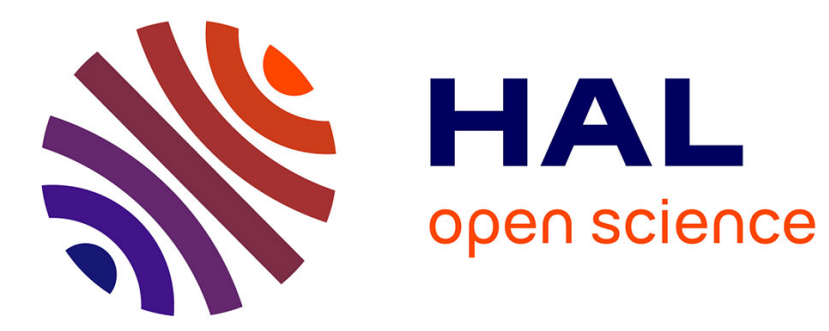

\title{
Coherent thermal infrared emission by two-dimensional silicon carbide gratings
}

Christophe Arnold, François Marquier, Moises Garin, Fabrice Pardo, Stéphane Collin, Nathalie Bardou, Jean-Luc Pelouard, Jean-Jacques Greffet

\section{To cite this version:}

Christophe Arnold, François Marquier, Moises Garin, Fabrice Pardo, Stéphane Collin, et al.. Coherent thermal infrared emission by two-dimensional silicon carbide gratings. Physical Review B: Condensed Matter and Materials Physics (1998-2015), 2012, 86 (3), pp.035316. 10.1103/PhysRevB.86.035316 . hal-00751541

\section{HAL Id: hal-00751541 \\ https://hal-iogs.archives-ouvertes.fr/hal-00751541}

Submitted on 19 Feb 2013

HAL is a multi-disciplinary open access archive for the deposit and dissemination of scientific research documents, whether they are published or not. The documents may come from teaching and research institutions in France or abroad, or from public or private research centers.
L'archive ouverte pluridisciplinaire HAL, est destinée au dépôt et à la diffusion de documents scientifiques de niveau recherche, publiés ou non, émanant des établissements d'enseignement et de recherche français ou étrangers, des laboratoires publics ou privés. 


\title{
Coherent thermal infrared emission by two-dimensional silicon carbide gratings
}

\author{
Christophe Arnold, ${ }^{1}$ François Marquier, ${ }^{2}$ Moisés Garin, ${ }^{3}$ Fabrice Pardo, ${ }^{1}$ Stéphane Collin, ${ }^{1}$ Nathalie Bardou, ${ }^{1}$ \\ Jean-Luc Pelouard, ${ }^{1}$ and Jean-Jacques Greffet ${ }^{2}$ \\ ${ }^{1}$ Laboratoire de Photonique et de Nanostructures, Centre National de la Recherche Scientifique, 91460 Marcoussis, France \\ ${ }^{2}$ Laboratoire Charles Fabry de l'Institut d'Optique, Centre National de la Recherche Scientifique, Campus Polytechnique, \\ RD 128, 91127 Palaiseau Cedex, France \\ ${ }^{3}$ Centro de Tecnologías Físicas. Unid. asoc. ICMM/CSIC-UPV Universitat Politècnica de València, Av. Tarongers s/n, 46022 València, Spain
}

(Received 25 January 2012; revised manuscript received 20 April 2012; published 17 July 2012)

\begin{abstract}
The thermal emission of cross-slit silicon carbide grating is studied in the Restrahlen region over all emission angles. We show experimentally that the thermal excitation of surface-phonon polaritons on the surface of 2D grating allows us to get a high emissivity in both polarizations, which is collimated in $p$ polarization for a specific wavelength determined by the periodicity of the grating. We also show numerically that $2 \mathrm{D}$ gratings optimized to efficiently out-couple thermally excited surface-phonon polaritons of the flat part of the dispersion relation can have a high efficiency for all emission directions for both polarizations.
\end{abstract}

DOI: 10.1103/PhysRevB.86.035316

PACS number(s): 44.40.+a, 71.36.+c, 63.22.-m

\section{INTRODUCTION}

Thermal radiation originates from the totally uncorrelated process of spontaneous emission in matter in local thermal equilibrium. In spite of the randomness involved in the mechanism of emission, it has been shown that some thermal sources could present high spatial coherence in the near field. ${ }^{1,2}$ This spatial coherence can be used to design highly directional sources with angular divergence comparable to lasers at the same wavelength ${ }^{3-5}$ or to strongly enhance or inhibit thermal radiation. ${ }^{6,7}$ Moreover, modern thermal emitters have shown that narrow-band thermal emission can be obtained far from the broad emission spectrum of conventional thermal sources. ${ }^{8}$

Modification of thermal emission has numerous potential applications: controlling the emission direction for local heating or the emission spectrum for thermophotovoltaic applications ${ }^{8-13}$ and spectroscopy. ${ }^{14}$ Production of infrared thermal sources with wavelengths of emission corresponding to specific absorption lines of gases could be useful for gas-sensing applications, for instance. ${ }^{12}$ Radiation cooling by enhancing the emissivity of a device is another original potential application. ${ }^{15}$

Various coherent thermal sources have been studied, but their principle is similar: The idea is to thermally excite electromagnetic modes having radiation losses. Recently, Schuller et al. have demonstrated narrow-band thermal emission by optical antenna. ${ }^{16}$ The use of metamaterials offers also a way to engineer thermal emission. Based on this principle, emitters presenting one or two wavelengths of emission have been obtained. $^{8}$

A way to get directional radiation is to thermally excite delocalized modes. In that case, the decay length characteristic of the mode propagation along the interface determines the spatial coherence length and the directionality of the source: The larger the spatial coherence length, the more directional the source. Sources based on excitation of guided modes have already shown spatial coherent thermal emission. ${ }^{17}$ It is also possible to use multilayer systems that act as wave-vector filters to generate spatial coherence. ${ }^{18,19}$ Similar effects have also been obtained with thermal sources having coupled resonant cavities. ${ }^{20}$
Surface waves are electromagnetic modes which can propagate along interfaces over long distances. This makes them natural candidates for the achievement of high coherent thermal sources. Structuring matter at the wavelength scale in order to couple surface waves to free-space plane waves has already been used to get directional thermal emission, mainly with 1D gratings. ${ }^{3,4} \mathrm{~A}$ few articles deal with the thermal emission of 2D metallic gratings assisted by surface-plasmon polaritons, ${ }^{21,22}$ but the radiation profiles are usually not investigated for all directions of emission. In this paper, the thermal radiation of silicon carbide cross-slit gratings is investigated over all emission angles for both linear polarizations. We show how the double periodicity of the gratings explain their high emissivity and their emission profiles by use of efficient diffraction of the thermally excited surface-phonons polaritons. Interestingly, we demonstrate experimentally that the emission is collimated in $p$ polarization in the direction perpendicular to the surface. Finally, we show numerically that it is possible to get an emissivity close to $100 \%$ for all emission angles by out-coupling the thermally excited surface-phonon polaritons with high wave vectors.

\section{COUPLING THE THERMALLY EXCITED SURFACE WAVES TO FREE SPACE}

Surface waves are supported by materials which have a negative real part of the dielectric constant. Metals are well known to support surface waves called surface-plasmon polaritons. Polar materials behave like metals in a small frequency domain, the Restrahlen region, where they support interface modes called surface-phonon polaritons (SPPs). Thus, such waves can exist on a $\mathrm{SiC}$ plane interface between 10.6 and $12.6 \mu \mathrm{m}$. Notice that this material is commonly used as thermal source because of its high melting point $(3000 \mathrm{~K})$.

In this paper, we study the emissivity of cross-slit $\mathrm{SiC}$ gratings. Several authors reported emissivity enhancement of $2 \mathrm{D}$ hole gratings by the excitation of surface plasmons, ${ }^{21-25}$ but emissivity of 2D polar crystal gratings assisted by surfacephonon polaritons has not been investigated. As Marquier et al. showed on 1D lamellar SiC gratings, ${ }^{26}$ the study of 
(a)
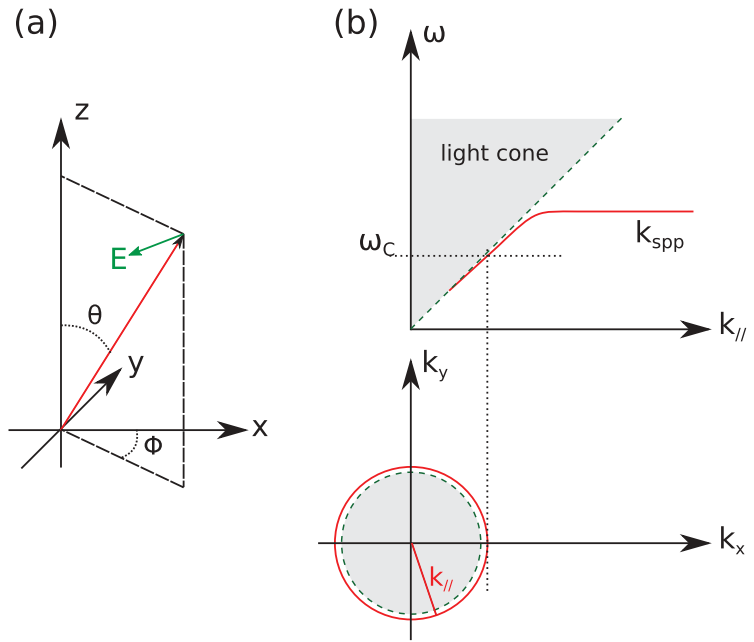

FIG. 1. (Color online) (a) Spherical coordinate system used in this paper. (b) Dispersion relation of the surface-phonon polaritons and its isofrequency projection on the $\left(\mathbf{k}_{\mathbf{x}}, \mathbf{k}_{\mathbf{y}}\right)$ plane; the gray areas represent the propagating waves in air.

the emission of such thermal sources cannot be limited to the plane perpendicular to the grooves, given that the profile and the polarization features of the thermal emission can be complex due to the coupling with SPPs.

Here we show that the thermal emission of a properly designed 2D grating can be concentrated in a collimated beam for a given wavelength in $p$ polarization. To describe the emissivity for all emission directions, we use a system of spherical coordinates [see Fig. 1(a)]. The azimuthal angle is given by the angle $\phi$ and $\theta$ is the angle between the emission wave vector and the surface normal $\vec{e}_{z}$. As shown in Fig. 1(b), the isofrequency projection of the surface wave dispersion relation of the unstructured surface on the $\left(\mathbf{k}_{\mathbf{x}}, \mathbf{k}_{\mathbf{y}}\right)$ plane is represented by a circle. This circle has a radius equal to the tangential component of the SPP wave vector $\left|\vec{k}_{\text {spp }}\right|$. Consider now a $2 \mathrm{D}$ grating with its double periodicity $\Lambda_{x}$ and $\Lambda_{y}$ along the axes $x$ and $y$. The dispersion relation in that case is represented by a lattice of circles of radius $\left|\vec{k}_{\mathrm{spp}}\right|$ centered on $2 \pi p / \Lambda_{x} \vec{e}_{x}+2 \pi q / \Lambda_{y} \vec{e}_{y}$, where $p$ and $q$ are relative integers ([Fig. 2(a)]. It is, hence, possible that a part of these circles lies inside the cross section of the light cone at this wavelength (gray area in the figure). We see that if we consider only this condition on the wave vectors, i.e., the phase matching, the coupling between surface waves and outgoing waves takes place along arcs of circle in the light cone. We point out that we simplified Fig. 2 by neglecting the higher diffraction orders. In fact, it appears in our simulation (not shown) that the coupling of these higher orders with SPPs is very low in our structure, which validates not considering them.

\section{NUMERICAL CALCULATIONS OF THE EMISSIVITY}

The grating under study is depicted in Fig. 2. The two periods are equal and chosen such that SPPs which propagate along $\vec{e}_{x},-\vec{e}_{x}, \vec{e}_{y}$, and $-\vec{e}_{y}$ couple with the wave propagating perpendicularly to the surface, at a wavelength near $12 \mu \mathrm{m}$ $\left(\Lambda=\Lambda_{x}=\Lambda_{y}=11.5 \mu \mathrm{m}\right)$. (a)

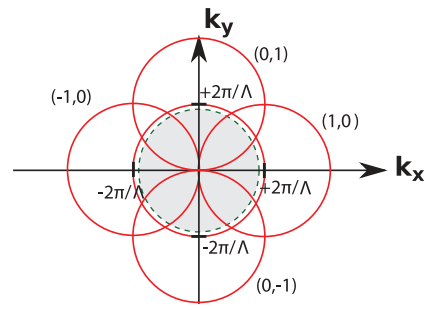

(b)

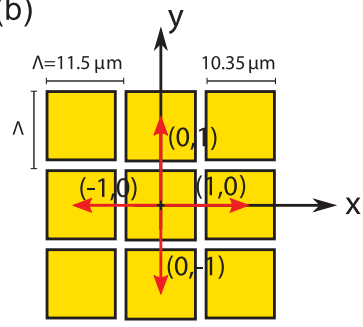

FIG. 2. (Color online) (a) Isofrequency contour of the SPP dispersion relation on the surface of a 2D shallow grating. (b) Scheme of the studied grating. The arrows represent the wave vectors of SPP which are coupled with the wave propagating perpendicularly to the surface.

For an opaque substrate, Kirchhoff's laws stipulate that emissivity is equal to $1-R, R$ being the reflectivity of the sample. ${ }^{27}$ We have calculated the reflectivity of this grating and, therefore, its emissivity by using a code based on the rigorous coupled wave analysis method. ${ }^{28,29}$ According to Palik, ${ }^{30}$ the dielectric constant of the $6 \mathrm{H} \mathrm{SiC}$, the material that we used here, can be considered as isotropic. We used a Lorentz model whose parameters are given by Spitzer et al. ${ }^{31}$ to calculate the dielectric constant of $\mathrm{SiC}$ at room temperature,

$$
\epsilon=\epsilon_{\infty}\left(1+\frac{\omega_{L}^{2}-\omega_{T}^{2}}{\omega_{T}^{2}-\omega^{2}-i \Gamma \omega}\right),
$$

with $\omega_{L}=969 \mathrm{~cm}^{-1}, \omega_{T}=793 \mathrm{~cm}^{-1}, \Gamma=4.76 \mathrm{~cm}^{-1}$, and $\epsilon_{\infty}=6.7$.

Figure 3 shows the calculated emissivity of the grating in $p$ (magnetic field parallel to the surface) [Fig. 3(a)] and $s$ (electric field parallel to the surface) [Fig. 3(b)] polarizations in the whole light cone at $12.1 \mu \mathrm{m}$ of wavelength. The two periods are $11.5 \mu \mathrm{m}$, the groove height is $700 \mathrm{~nm}$, and the filling fractions (ratio between ridge and period) $f_{x}=f_{y}=90 \%$.

Let us consider, first, the emission in the $s$ polarization depicted on Fig. 3(b). The enhancement of emissivity follows arcs of circle as expected by the phase matching condition between SPPs and outgoing waves [Fig. 2(a)]. It is interesting to notice the high emissivity in this polarization, to $97 \%$ at the maximum. It reveals an efficient coupling between surfaces waves and outgoing $s$-polarized waves. This efficient coupling is not intuitive given that only $p$-polarized waves are coupled to surface plasmons for lamellar grating in the plane perpendicular to the grating lines. However, as soon as out-of-plane diffraction is considered, surface plasmons can be coupled to $s$-polarized waves.

At the same wavelength, the emissivity in $p$ polarization is highly directional. We obtained a collimated thermal radiation beam, with a high emissivity, to $97 \%$ and a square profile [see the inset in Fig. 3(a)]. We can notice that there is little enhancement of emissivity along the arcs of circle given by the phase matching, revealing no efficient coupling of SPP with outgoing waves. This can be explained using a polarization matching condition in the coupling between SPPs and propagating waves. Figure 4(a) [respectively, Fig. 4(b)] represents the electric and magnetic fields of an outgoing $p$-polarized (respectively, $s$-polarized) wave, as well as the 

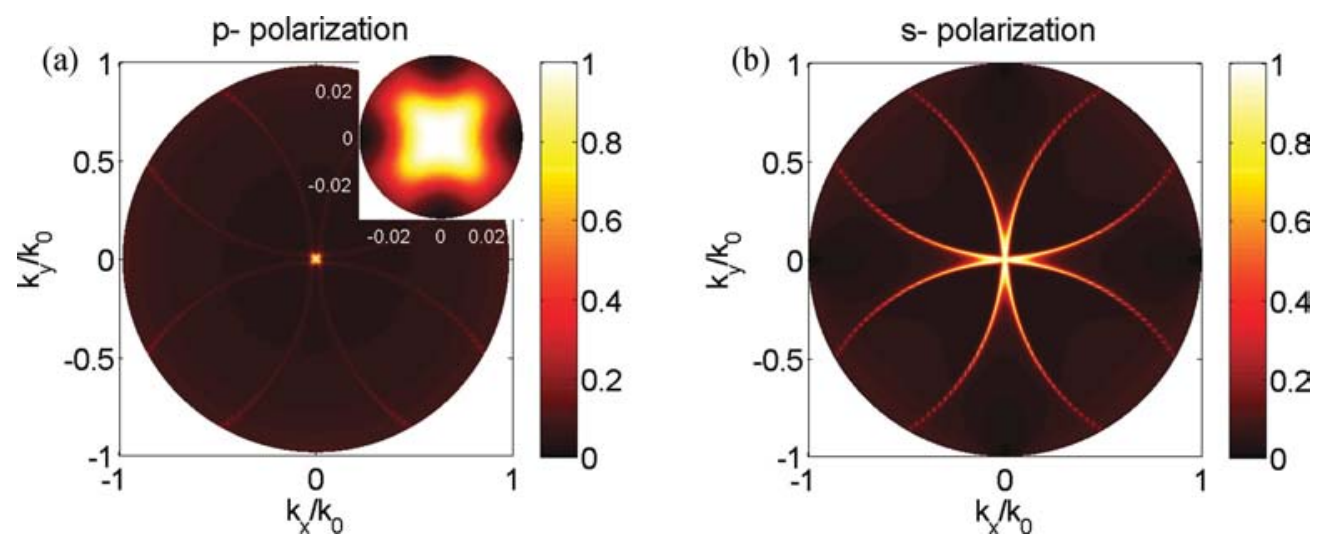

FIG. 3. (Color online) Polar representation of the calculated emissivity at $\lambda=12.1 \mu \mathrm{m}$ in both $p$ (a) and $s$ polarizations (b) of a cross-slit $\mathrm{SiC}$ grating. The inset is the emissivity in $p$ polarization close to direction perpendicular to the surface. The parameters of this grating are $\Lambda_{x}=\Lambda_{y}=11.5 \mu \mathrm{m}, h=700 \mathrm{~nm}$, and $f_{x}=f_{y}=90 \%$.

electromagnetic components of the SPP wave which is phase matched with this outgoing wave. In $p$ polarization, we can notice the mismatch between the polarizations of these two waves. Electric and magnetic fields of the two waves are nearly orthogonal with one another. This does not allow an efficient coupling. ${ }^{15}$ This polarization mismatch explains the weak coupling with surface waves and so the weak emissivity in this polarization along arcs of circle. Conversely, there is good polarization matching between the outgoing wave and the SPP for $s$-polarization emission; thus, the coupling is possible between them. This explains the high emissivity in this polarization.

We point out that this collimation is obtained only around this specific wavelength in a range of wavelength of $140 \mathrm{~nm}$. For a different wavelength, the grating emits a high emissivity along arcs of circle determined by the phase matching condition.

\section{FABRICATION AND EMISSIVITY MEASUREMENTS}

We fabricated 2D SiC gratings by use of anisotropic dry etching of a $6 \mathrm{H}$ on-axis crystalline $\mathrm{SiC}$ substrate. The different fabrication steps are depicted in Figs. 5(a)-5(c). A crossline pattern was obtained by electron-beam lithography in a
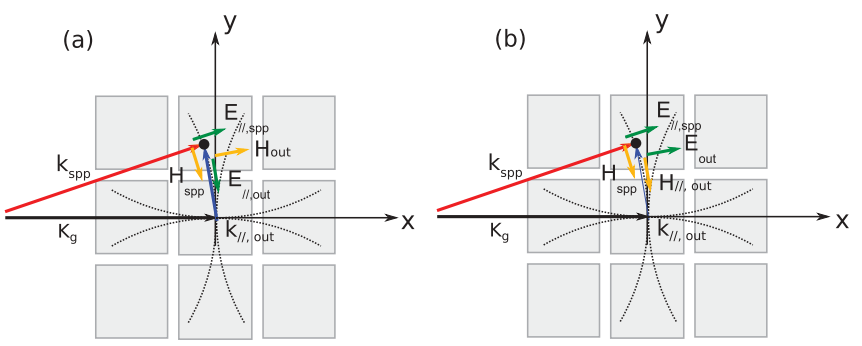

FIG. 4. (Color online) The two schemes represent the components parallel to the interface of the wave vectors of the electric and magnetic fields for the outgoing wave and for the coupled SPP in $p$ (a) and $s$ polarization (b). In this example, the outgoing wave and the SPP are coupled by the $(-1,0)$ order of the grating, and their wave vectors follow the phase matching condition $\mathbf{k}_{/ /, \text {out }}=\mathbf{k}_{\mathrm{spp}}-\mathbf{K}_{\mathrm{g}}$, with $K_{g}=\frac{2 \pi}{\Lambda} \mathbf{e}_{\mathbf{x}}$. 700-nm negative resist (maN-2410). After resist development, we deposited, by sputtering, a 100-nm-thick layer of nickel in order to get a mask after the lift-off of the resist [Fig. 5(a)]. The grooves are obtained after reactive ionic etching of the $\mathrm{SiC}$ [Fig. 5(b)]. The final grating is obtained after chemical etching of nickel in a $\mathrm{HNO}_{3}$ solution [Fig. 5(c)]. In order not to limit the spatial coherence of our source, we fabricated the grating on a large surface of $1 \mathrm{~cm}^{2}$. Figure $5(\mathrm{~d})$ is a scanning electron microscope image of a fabricated grating. The slopes of the slits of the fabricated gratings are steep (near $80 \mathrm{deg}$ ) and their bottom is smooth enough compared to the wavelength. The roughness of the surface visible on the image comes from the manufacturer polishing, but it is also much smaller than the wavelength.

We measured the emissivity of fabricated gratings with a Fourier transform spectrometer (FTIR) (Bomem DA08). The spectral resolution is $1 \mathrm{~cm}^{-1}$. Angular resolution, around $0.5 \mathrm{deg}$, is obtained using an aperture in Fourier's plane of a spherical mirror. The experimental setup to measure the emitted flux is described in detail in Ref. 6. We normalized the measurements with a standard radiometric calibration following Ref. 32. This method consists of measuring the flux of a reference blackbody at two different temperatures. Thermal radiation of the room and of the spectrometer itself (a)

(b)

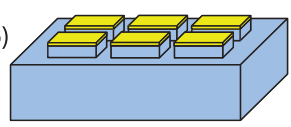

(c)

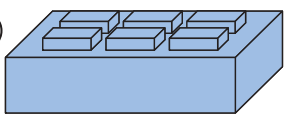

(d)

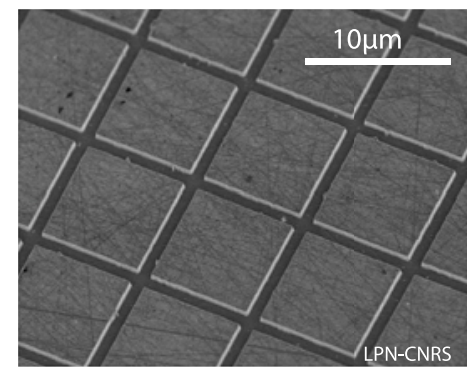

FIG. 5. (Color online) [(a)-(c)] Schemes of the fabrication steps of the grating. (a) Mask of nickel obtained by electronic lithography on a SiC substrate (200 $\mu \mathrm{m}$ thick). (b) Reactive ionic etching of the SiC. (c) Final grating after chemical etching of the nickel mask in a $\mathrm{HNO}_{3}$ solution. (d) Scanning electron microscope image of the sample. 


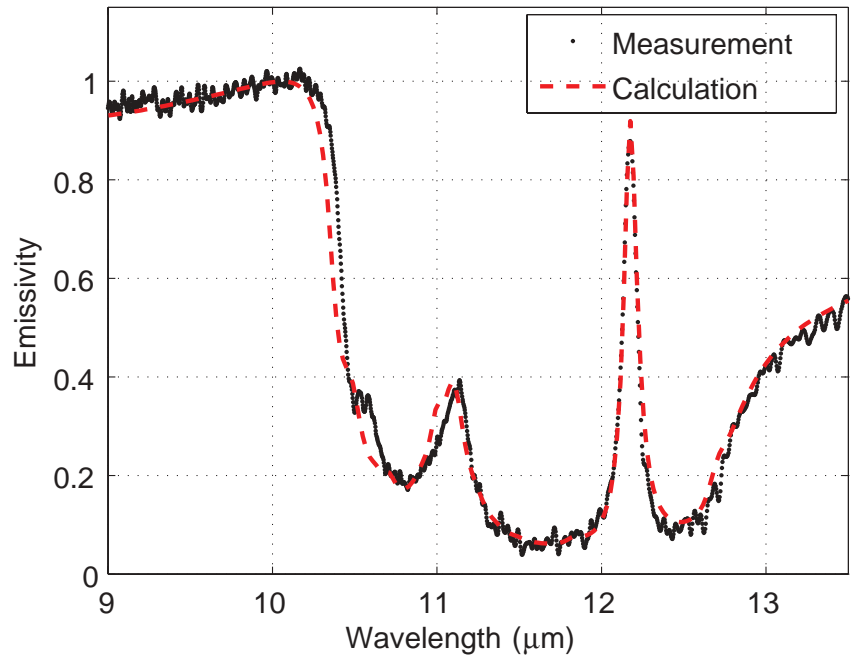

FIG. 6. (Color online) Measurement (dots) and calculation (dashed line) of the emissivity spectrum in the direction normal to the surface. Parameters of the grating: $\Lambda_{x}=\Lambda_{y}=11.5 \mu \mathrm{m}$, $f_{x}=f_{y}=0.88$, and $h=700 \mathrm{~nm}$.

are important in this wavelength range. To minimize phaseinduced errors coming from these parasite fluxes, we subtract them from the measurements of the sample radiation before performing the Fourier transform. ${ }^{33}$

The dotted curve of Fig. 6 shows the measured emissivity of a sample for wavelengths between 9 and $13.5 \mu \mathrm{m}$ in the direction normal to the surface. The analyzer is parallel to one set of grooves. The sample was maintained at a temperature of $400^{\circ} \mathrm{C}$. In order to obtain the emissivity spectrum, we needed to know the exact value of the sample temperature. We deduced it using the Christiansen wavelength, near $10.1 \mu \mathrm{m}$ : At this wavelength, the refractive index is close to 1, so the reflectivity is close to 0 , and, following Kirchhoff's laws, the emissivity tends to be 1 . We therefore can precisely deduce the experimental temperature by comparison of the measured thermal emission with Planck's spectrum at this wavelength.

The first peak near $11.1 \mu \mathrm{m}$ is due to the excitation of the surface-phonon polaritons on the flat part of the dispersion relation [see Fig. 1(b)]. We will see in Sec. V that optimizing a grating for this particular wavelength allows us to get a highly isotropic thermal source. We optimized the grating to have a high emissivity at $12.1 \mu \mathrm{m}$. Close to this wavelength $(12.18 \mu \mathrm{m})$, we indeed measured a narrow peak with a width at half-maximum of $100 \mathrm{~nm}$ and an emissivity close to $90 \%$. The dashed curve is the calculated emissivity of this grating. For the calculations, we used the grating filling fraction measured with an electron microscope $(88 \%)$, a thickness of $700 \mathrm{~nm}$, and the two periods equal to $11.5 \mu \mathrm{m}$. To take into account the influence of the sample temperature on the optical properties in our simulations, we varied the parameters of the Lorentz model by less than $1 \%$ compared to those given by Spitzer et al. for $\mathrm{SiC}$ at room temperature, except losses ( $\Gamma$ coefficient $)$, which we increased by $45 \%$ in agreement with Ref. 6 . The parameters used for the fit are $\omega_{L}=966 \mathrm{~cm}^{-1}, \omega_{T}=786 \mathrm{~cm}^{-1}, \Gamma=$ $7.5 \mathrm{~cm}^{-1}$, and $\epsilon_{\infty}=6.75$.

In order to confirm that the thermal radiation is collimated in $p$ polarization at this wavelength, we measured the emissivity in two planes of emission with $\phi=0$ and $45 \mathrm{deg}$. These two planes are shown in Fig. 7 by the blue and red dashed lines, respectively. Figure 7(b) gives the measured (cross and square markers) and calculated (dashed lines) emissivity in these directions. We find a good agreement between data and theory. The angular width of the emissivity peak is equal to $3.9 \mathrm{deg}$.

We also measured the emissivity in $s$ polarization. We measured it along two sections of the light cone which are indicated by the lines on Fig. 8. The dashed line corresponds to measurements with $\phi=0 \mathrm{deg}$ and we scan the angle $\theta$, and the dotted line corresponds to measurements with $\theta=20 \mathrm{deg}$ and we scan the angle $\phi$. Calculated (lines) and measured (markers) emissivity are shown on Figs. 8(b) and 8(c). Note that the angular width is broader in this polarization (around $19 \mathrm{deg}$ when $\phi=0 \mathrm{deg}$ ).

\section{ISOTROPIC EMISSION BY THE THERMAL EXCITATION OF SURFACE-PHONON POLARITONS}

In Ref. 26, authors show that a 1D grating presents a high isotropic emissivity when the surface-phonon polaritons with a large wave vector (flat part of the dispersion relation) are
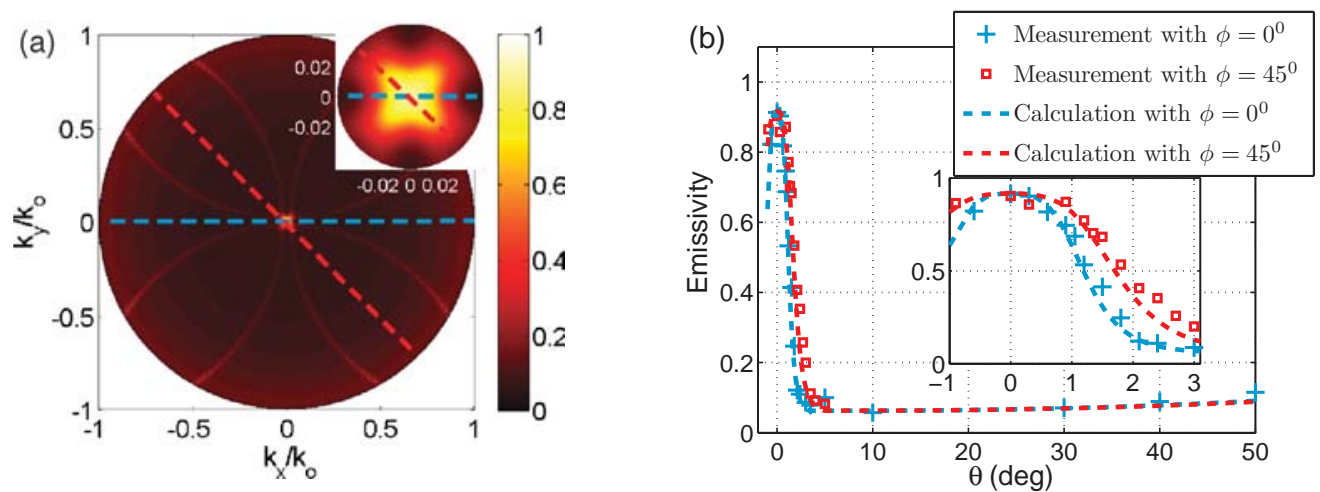

FIG. 7. (Color online) (a) Polar representation of the calculated emissivity at $\lambda=12.18 \mu \mathrm{m}$ in $p$ polarization. The blue and red lines represent the directions for which emissivity were measured. (b) Emissivity as a function of $\theta$ at $\lambda=12.18 \mu \mathrm{m}$, for two azimuthal angles, $\phi=0 \mathrm{deg}$ (blue) and $\phi=45 \mathrm{deg}$ (red) in $p$ polarization. Cross and square markers represent the measurements and the lines the calculations. 

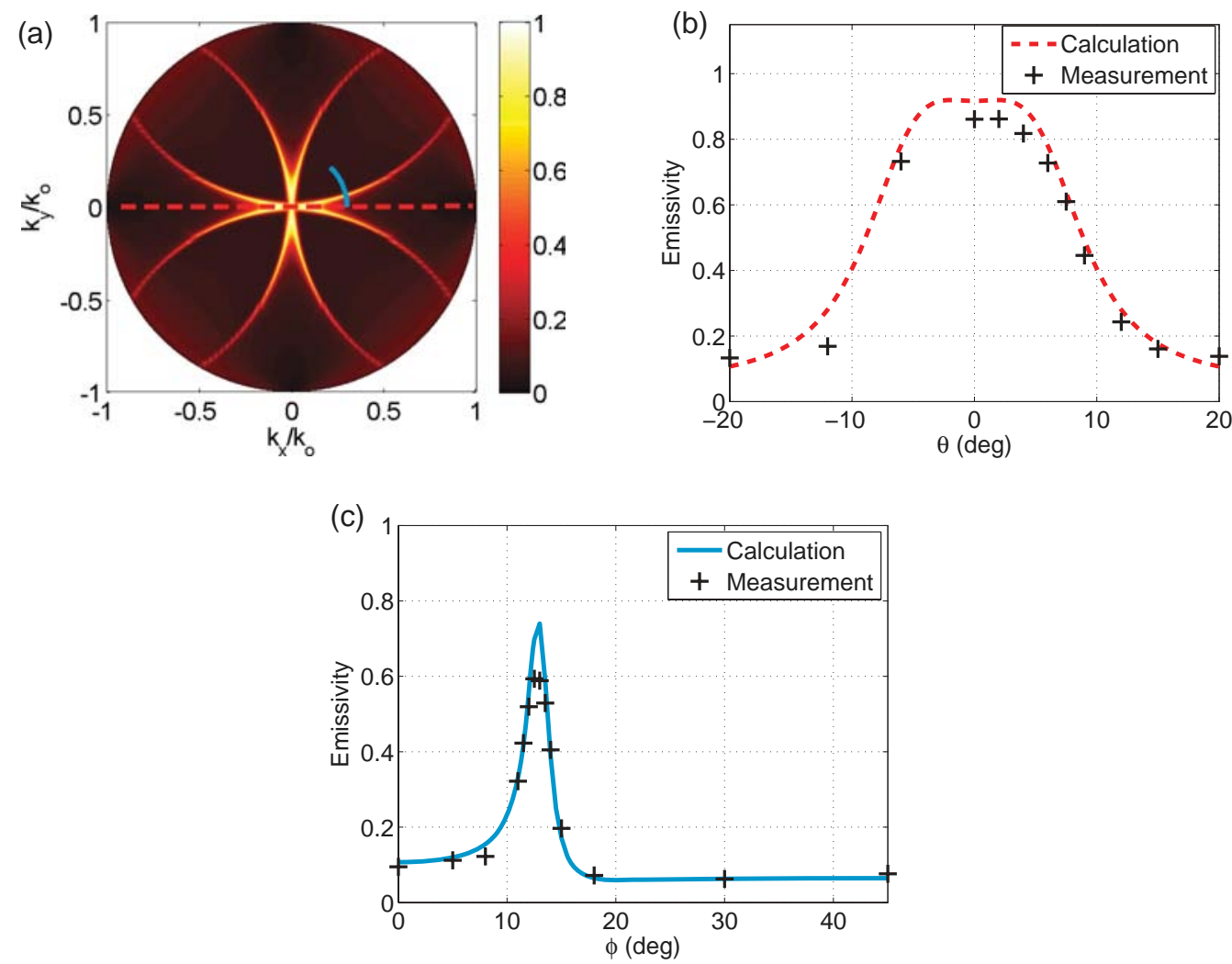

FIG. 8. (Color online) (a) Polar representation of the calculated emissivity at $\lambda=12.18 \mu \mathrm{m}$ in $s$ polarizations. The blue and red lines represent the directions for which emissivity were measured. (b) Measured (markers) and calculated (line) emissivity at $\lambda=12.18 \mu \mathrm{m}$ as a function of $\theta$, with $\phi=0 \mathrm{deg}$, in $s$ polarization. (c) Measurement (markers) and calculation (dashed line) of the emissivity as a function of the azimuthal angle $\phi$, with an incident angle $\theta=20 \mathrm{deg}$, in $s$ polarization.

coupled with free space. The emission is no longer directional because the phase matching condition is fulfilled for any direction in the light cone. This is due to the fact that the dispersion relation is flat for this particular wavelength so any value of the wave vector is available. However, the unpolarized emissivity of this 1D grating was limited to $50 \%$, because the polarization matching condition was not fulfilled with a 1D grating for any direction. Here, we predict that 2D gratings allow an isotropic emission with an emissivity close to $100 \%$ over all emission angles. The double periodicity ensures that both phase and polarization matching conditions are fulfilled with at least one of the diffracted orders of the grating for any direction. The cross-slit grating that we optimized to efficiently out-couple SPPs on the flat part of the dispersion relation has its two periods of $3 \mu \mathrm{m}$, the filling fractions $f_{x}$ and $f_{x}$ of 0.5 and the groove depth of $750 \mathrm{~nm}$. Figure 9 shows the calculated emissivity for this grating in $p$ [Fig. 9(a)] and $s$ [Fig. 9(b)] polarizations, at $\lambda=11.2 \mu \mathrm{m}$. The thermal emission is isotropic for both polarizations, with an emissivity close to 1 . We point out that this isotropy is obtained only on the flat part of the dispersion relation of surface-phonon polaritons, for a range of wavelength comparable to the mode linewidth at this wavelength, which is around $200 \mathrm{~nm}$.
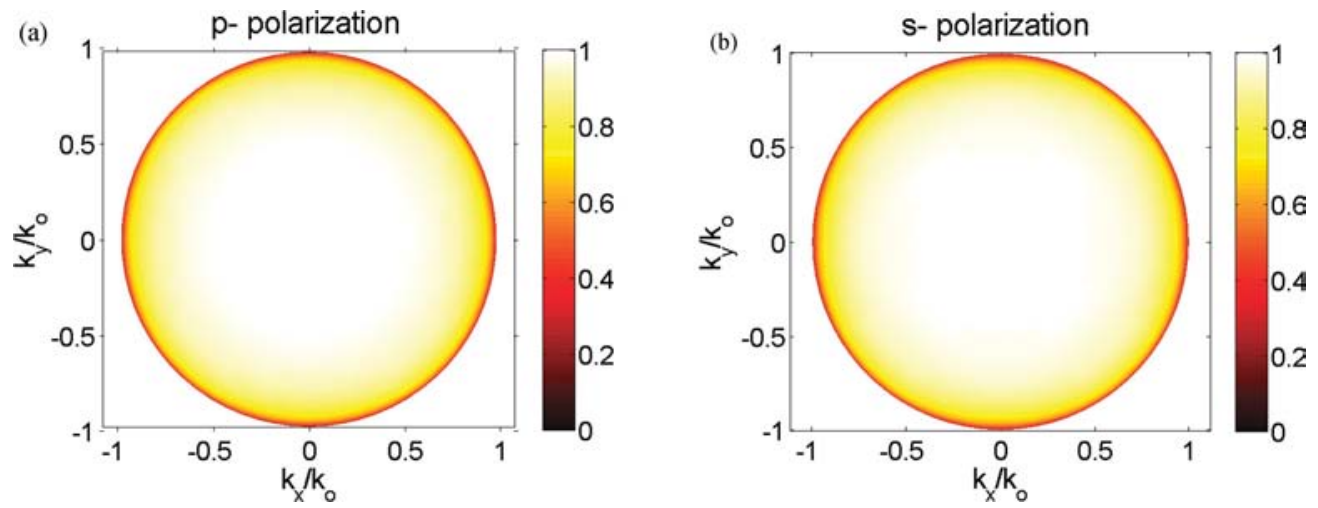

FIG. 9. (Color online) Calculated emissivity in $p$ (a) and $s$ (b) polarizations in the light cone at $\lambda=11.2 \mu \mathrm{m}$. 


\section{CONCLUSION}

In summary, we studied the emissivity of cross-slit SiC gratings for all emission directions for both linear polarizations. We optimized the grating design to efficiently emit in the direction normal to the surface for a given wavelength. We showed that the thermal radiation of our structure presents a highly collimated beam with an angular width around $3.9 \mathrm{deg}$ and an emissivity close to $90 \%$ in $p$ polarization for a specific wavelength determined by the periodicity of the grating. We also measured a high emissivity in $s$ polarization, although surface-phonon polaritons are $p$ polarized. The coupling between surface waves and propagating waves mediated by the $2 \mathrm{D}$ grating is efficient provided that phase and polarization matching conditions are fulfilled. We explain the emission profile of the 2D grating with these simple rules. We, finally, showed numerically that the thermal excitation of surface waves with large wave vectors and their coupling with a $2 \mathrm{D}$ grating allows us to obtain a highly isotropic thermal source with emissivity close to 1 . It is worth noting that the design rules of directional sources can be used with metallic thermal sources taking advantage of surface-plasmon polaritons.

\section{ACKNOWLEDGMENTS}

C.A. acknowledges financial support from the French Ministry of Defense through a grant from the Délégation Générale pour l'Armement (DGA).
${ }^{1}$ R. Carminati and J.-J. Greffet, Phys. Rev. Lett. 82, 1660 (1999).

${ }^{2}$ A. Joulain, J.-P. Mulet, F. Marquier, R. Carminati, and J.-J. Greffet, Surf. Sci. Rep. 57, 59 (2005).

${ }^{3}$ J.-J. Greffet, R. Carminati, K. Joulain, J.-P. Mulet, S. Mainguy, and Y. Chen, Nature 416, 61 (2002).

${ }^{4}$ M. Laroche, C. Arnold, F. Marquier, R. Carminati, J.-J. Greffet, S. Collin, N. Bardou, and J.-L. Pelouard, Opt. Lett. 30, 2623 (2005).

${ }^{5}$ G. Biener, N. Dahan, A. Niv, V. Kleiner, and E. Hasman, Appl. Phys. Lett. 92, 081913 (2008).

${ }^{6}$ F. Marquier, K. Joulain, J.-P. Mulet, R. Carminati, J.-J. Greffet, and Y. Chen, Phys. Rev. B 69, 155412 (2004).

${ }^{7}$ S.-Y. Lin, J. G. Fleming, E. Chow, J. Bur, K. K. Choi, and A. Goldberg, Phys. Rev. B 62, R2243 (2000).

${ }^{8}$ X. Liu, T. Tyler, T. Starr, A. F. Starr, N. M. Jokerst, and W. J. Padilla, Phys. Rev. Lett. 107, 045901 (2011).

${ }^{9}$ H. Sai and H. Yugami, Appl. Phys. Lett. 85, 3399 (2004).

${ }^{10}$ F. O’Sullivan, I. Celanovic, N. Jovanovic, J. Kassakian, S. Akiyama, and K. Wada, J. Appl. Phys. 97, 033529 (2005).

${ }^{11}$ M. Laroche, R. Carminati, and J.-J. Greffet, J. App. Phys. 100, 063704 (2006).

${ }^{12}$ J.-J. Greffet, Nature 478, 191 (2011).

${ }^{13}$ P. Ben-Abdallah, J. Opt. Soc. Am. A 21, 1368 (2004).

${ }^{14}$ I. Puscasu and W. L. Schaich, Appl. Phys. Lett. 92, 233102 (2008).

${ }^{15}$ F. Marquier, M. Laroche, R. Carminati, and J.-J. Greffet, J. Heat Trans. 129, 11 (2007).

${ }^{16}$ J. A. Schuller, T. Taubner, and M. L. Brongersma, Nat. Photon. 3, 658 (2009).

${ }^{17}$ K. Joulain and A. Loizeau, J. Quant. Spectrosc. Radiat. Transf. 104, 208 (2007).
${ }^{18}$ B. J. Lee, C. J. Fu, and Z. M. Zhang, Appl. Phys. Lett. 87, 071904 (2005).

${ }^{19}$ J. Drevillon and P. Ben-Abdallah, J. Appl. Phys. 102, 114305 (2007).

${ }^{20}$ N. Dahan, A. Niv, G. Biener, Y. Gorodetski, V. Kleiner, and E. Hasman, Phys. Rev. B 76, 045427 (2007).

${ }^{21}$ I. Puscasu, M. Pralle, M. McNeal, J. Daly, A. Greenwald, E. Johnson, R. Biswas, and C. G. Ding, J. Appl. Phys. 98, 013531 (2005).

${ }^{22}$ M.-W. Tsai, T.-H. Chuang, C.-Y. Meng, Y.-T. Chang, and S.-C. Lee, Appl. Phys. Lett. 89, 173116 (2006).

${ }^{23}$ S. Maruyama, T. Kashiwa, H. Yugami, and M. Esashi, Appl. Phys. Lett. 79, 1393 (2001).

${ }^{24}$ H. Sai, H. Yugami, Y. Akiyama, Y. Kanamori, and K. Hane, J. Opt. Soc. Am. A 18, 1471 (2001).

${ }^{25}$ R. Biswas, C. G. Ding, I. Puscasu, M. Pralle, M. McNeal, J. Daly, A. Greenwald, and E. Johnson, Phys. Rev. B 74, 045107 (2006).

${ }^{26}$ F. Marquier, C. Arnold, M. Laroche, J. J. Greffet, and Y. Chen, Opt. Express 16, 5305 (2008).

${ }^{27}$ J.-J. Greffet and M. Nieto-Vesperinas, J. Opt. Soc. Am. A 15, 2735 (1998).

${ }^{28}$ L. Li, J. Opt. Soc. Am. A 14, 2758 (1997).

${ }^{29}$ P. Lalanne and G. M. Morris, J. Opt. Soc. Am. A 13, 779 (1996).

${ }^{30}$ E. D. Palik, Handbook of Optical Constants of Solids (Academic Press, New York, 1985).

${ }^{31}$ W. G. Spitzer, D. Kleinman, and D. Walsh, Phys. Rev. 113, 127 (1959).

${ }^{32}$ H. E. Revercomb, H. Buijs, H. B. Howell, D. D. LaPorte, W. L. Smith, and L. A. Sromovsky, Appl. Opt. 27, 3210 (1988).

${ }^{33}$ B. Carli, L. Palchetti, and P. Raspollini, Appl. Opt. 38, 7475 (1999). 